\title{
Ecotoxicidade do 2,4-D a oligoquetas em função do tipo de solo ${ }^{1}$
}

\author{
Bioassays of 2,4-D with earthworms in different soil classes
}

\author{
Daniel Gomes da Costa ${ }^{2}$; Tácio Mauro Pereira de Campos $^{3}$; Ricardo Gonçalves Cesar ${ }^{4}$; Zuleica \\ Carmen Castilhos ${ }^{5}$; Bianca Carolina Resende Carneiro da Rocha ${ }^{6}$
}

Resumo - Apesar de classificado como extremamente tóxico, com possível ação neurotóxica, caráter teratogênico, e possivelmente carcinogênico, o herbicida 2,4-D ainda é bastante utilizado na agricultura, sendo seus efeitos ao meio ambiente constantemente estudados. O presente trabalho propõe a avaliação da ecotoxicidade deste herbicida em bioensaio com oligoquetas (Eisenia andrei) em quatro tipos de solos. Para tanto, ensaios de toxicidade aguda foram realizados com um solo sedimentar, dois coluvionares e um residual, previamente contaminados com soluções de concentração agronômica de 2,4-D. Não foram observados níveis significativos de mortalidade de animais, sugerindo baixa toxicidade aguda dos solos para os oligoquetas edáficos. Porém, os resultados revelaram que os menores níveis de toxicidade foram atrelados ao aspecto textural e a alta microporosidade, sendo o maior potencial tóxico atribuído ao solo arenoso formado basicamente por quartzo. Esses resultados deverão auxiliar o estabelecimento futuro de valores de referência tóxica capazes de refletir as ocorrências pedológicas brasileiras, subsidiando futuras avaliações de risco ecológico e a tomada de decisão em medidas de saúde ambiental.

Palavras-chaves: bioensaios; herbicida; solos

Abstract - 2,4-D is a highly toxic herbicide, neurotoxic, teratogenic, possibly carcinogenic and well-known by its capacity of causing damages on human health and biota. This work proposes the assessment of 2,4-D toxicity levels in four Brazilian soil classes, using bioassays with earthworms (Eisenia andrei). Acute toxicity tests were performed with one sedimentary, two colluvial and one residual soils previously infected with solutions of agronomical concentration. No significant levels of mortality were observed, suggesting low acute toxicity to soil earthworms. However, the results revealed that lower levels of toxicity were linked to the textural appearance and high microporosity. The largest toxic potential was attributed to the quartz sandy soil. Such results may support the future establishment of toxic reference values able to reflect Brazilian pedological occurrences, subsiding future ecological risk assessments and decision-

\footnotetext{
${ }^{1}$ Recebido para publicação em 31/10/2015 e aceito em 04/12/2015.

2 Mestrando em Geotecnia da Pontifícia Universidade Católica do Rio de Janeiro, Centro Técnico-Científico, Departamento de Engenharia Civil. R. Marquês de São Vicente, 225 - Gávea, Rio de Janeiro - RJ, $22451-900$. <dgcdaniel@hotmail.com>

3 Professor da Pontifícia Universidade Católica do Rio de Janeiro, Centro Técnico-Científico, Departamento de Engenharia Civil.

4 Professor da Universidade Federal do Rio de Janeiro. Instituto de Geociências. Departamento de Geografia. Laboratório de Ecologia e Ecotoxicologia de Solos. Av. Athos da Silveira Ramos, 274. Prédio do Centro de Ciências Matemáticas e da Natureza, Bloco F - Ilha do Fundão, Rio de Janeiro - RJ, 21941-916.

5 Pesquisadora do Centro de Tecnologia Mineral, CETEM/MCTI. Coordenação de Processos Minerais/ Desenvolvimento Sustentável. Av. Pedro Calmon, 900. Cidade Universitária, Rio de Janeiro - RJ, 21941-908.

${ }^{6}$ Bolsista de IC do Centro de Tecnologia Mineral, CETEM/MCTI.
} 
making in actions of environmental health.

Keywords: bioassays; herbicide; soils

\section{Introdução}

O 2,4-D é um herbicida extremamente tóxico e conhecido pela capacidade de causar danos à saúde humana e ambiental. O seu emprego está relacionado à agricultura com a finalidade de controlar ervas em mais de 1500 produtos e em algumas formulações de herbicidas no Brasil e no exterior (Amarante Júnior et al., 2002). Em função das vias de degradação bióticas e abioticas, a persistência do 2,4-D e de seus resíduos podem durar de poucos dias a vários meses no solo e na água (Amarante Júnior et al., 2002; Holt et al., 2012; Gervais et al., 2008).

A maior parte dos estudos geoambientais em solos está baseada em análises químicas totais, extrações sequênciais, e/ou extrações seletivas de frações geoquímicas de elevado potencial de mobilidade e/ou biodisponibilidade. Porém, essas abordagens não levam em consideração o efeito sinérgico de contaminantes sobre os organismos. A abordagem ecotoxicológica pode complementar estes resultados por meio do monitoramento de efeitos letais, subletais e estimativa das frações biodisponíveis pela determinação dos teores de contaminantes em tecidos de organismos expostos (Straalen et al, 2005; Cesar et al, 2010).

Os oligoquetas têm sido largamente utilizados como organismos-teste em ensaios ecotoxicológicos (Nahmani et al., 2007; Carbonell et al., 2009; Moreira et al., 2008; Cesar et al., 2014). A escolha pela Eisenia andrei deve-se pela padronização internacional dos ensaios ecotoxicológicos (Nunes, 2010). As minhocas possuem grande importância ecológica, devido à sua abundância em solos tropicais e temperados, no seu papel na decomposição e na melhoria da textura dos solos, e por servirem como elo importante da cadeia trófica terrestre (Vijver et al., 2003). Além disso, seu emprego nos bioensaios se justifica pelo cultivo simples em laboratório, pela praticidade em manipulá-las durante os experimentos, por ingerirem grande quantidade de solo, pelo relativo curto ciclo de vida, por representarem mais de $90 \%$ da pedobiomassa, quando presente, pela possibilidade de serem expostas de diversas formas aos contaminantes e, por serem sensíveis à presença de agentes tóxicos (Liu et al., 2005, Nahmani et al., 2007, Hinton e Veiga, 2008).

O comportamento pedogeoquímico do 2,4-D depende, por exemplo, das propriedades físicas, químicas e mineralógicas do solo, tendo a textura, o $\mathrm{pH}$, mineralogia de argilas, e matéria orgânica papéis fundamentais neste processo (Amarante Júnior et al., 2002; Spadotto et al., 2003; Vivian et al., 2007; Queiroz, 2007). Essas propriedades são importantes na determinação dos níveis de mobilidade do herbicida, bem como de sua biodisponibilidade e toxicidade ambiental.

O presente trabalho trata do estudo da ecotoxicidade e da biodisponibilidade do 2,4-D em quatro classes de solos tropicais artificialmente contaminadas, utilizando testes ecotoxicológicos com oligoquetas (Eisenia andrei).

\section{Material e Métodos}

\section{Reagentes}

Padrão do herbicida 2,4-D ( $\geq 98 \%$ de pureza) da Sigma-Aldrich.

\section{Amostras dos solos}

Uma amostra de solo (denominado solo 1) foi retirada no campus da Universidade Federal Rural do Rio de Janeiro, situada em Seropédica. Outras duas coletas (solos 2 e 3) foram realizadas no campus avançado da PUCRio em Tinguá. A última coleta de solo (solo 4) aconteceu no município de Duque de Caxias, em uma rua vicinal, no $\mathrm{km} 12$ da 
Rodovia Washington Luiz. Os ensaios de caracterização física e mineralógica seguiram as recomendações da Associação Brasileira de Normas Técnicas (Tabela). Também foi realizada a difração de Raios-X em pó com a fração fina dos materiais para determinar a composição mineralógica do material. O solo 1 apresentou apenas quartzo em sua composição e o solo 2 apresentou quartzo e gibsita. A caulinita, gibsita, goetita e quatzo foram observadas no solo 3 , enquanto que, o solo 5 apresentou quartzo, goetita e calulinita.

Tabela 1. Características físico-químicas dos solos para avaliação da resposta dos oligoquetas após aplicação do 2,4-D.

\begin{tabular}{|c|c|c|c|c|c|}
\hline & & Solo 1 & Solo 2 & Solo 3 & Solo 4 \\
\hline \multirow{6}{*}{ 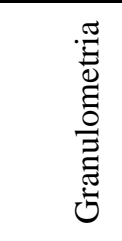 } & Pedregulho (\%) & 0,6 & 4,1 & 1,4 & 0,0 \\
\hline & Areia Grossa (\%) & 27,8 & 30,8 & 15,2 & 0,7 \\
\hline & Areia Média (\%) & 35,5 & 32,7 & 15,3 & 13,4 \\
\hline & Areia Fina (\%) & 25,5 & 18,3 & 6,6 & 22,3 \\
\hline & Silte (\%) & 8,5 & 10 & 9,8 & 49,7 \\
\hline & Argila (\%) & 2,3 & 4,2 & 51,7 & 13,9 \\
\hline \multicolumn{2}{|c|}{ Limite de Liquidez - LL (\%) } & - & - & 71,6 & - \\
\hline \multicolumn{2}{|c|}{ Limite de Plasticidade - LP (\%) } & - & - & 33,8 & - \\
\hline \multicolumn{2}{|c|}{ Indíce de Atividade $-\mathrm{I}_{\mathrm{a}}$} & - & - & 0,63 & - \\
\hline \multicolumn{2}{|c|}{ Teor de umidade natural - w $(\%)$} & 4,4 & 16,5 & 25,3 & 25,5 \\
\hline \multicolumn{2}{|c|}{ Porosidade $-\mathrm{n}(\%)$} & 35,77 & 57,77 & 55,99 & 61,84 \\
\hline \multicolumn{2}{|c|}{ Densidade in situ $-\rho\left(\mathrm{g} / \mathrm{cm}^{3}\right)$} & 1,77 & 1,45 & 1,46 & 1,32 \\
\hline \multicolumn{2}{|c|}{ Teor de matéria orgânica - MO (\%) } & 0,73 & 1,77 & 0,74 & 1,98 \\
\hline \multicolumn{2}{|l|}{$\mathrm{pH}$} & 4,74 & 4,27 & 3,89 & 3,55 \\
\hline
\end{tabular}

De acordo com o clássico triângulo de classificação textural, os solos 1, 2, 3 e 4 pertencem às seguintes classes texturais: areia, areia, argila e franco siltoso, respectivamente.

\section{Experimento}

A aplicação do 2,4-D nas amostras de solo foi feita manualmente de acordo com a quantidade agronômica maior indicada pelo MAPA (2003), sendo a dose do produto de $7 \%$ e volume de calda de 250 L.ha $^{-1}$ (i.e., $12,43 \mathrm{mg}$ de 2,4-D). Primeiramente o 2,4-D foi misturado ao volume de água destilada correspondente a $50 \%$ da capacidade de retenção de água de cada solo para depois ser aplicado as amostras de solo.

Foi realizado um estudo para avaliação do efeito tóxico agudo da concentração de 2,4D sobre o oligoqueta edáfico da Eisenia andrei nos quatro solos coletados e um solo artificial como controle. As características diferenciadas de cada solo deverão possibilitar o estudo da influência de suas propriedades sobre a biodisponibilidade e a ecotoxicidade do herbicida. A avaliação desta esteve fundamentada na comparação entre os níveis de sobrevivência e de biomassa obtidos com os solos puros (i.e., sem adição de herbicida) e os solos contaminados.

Os organismos-teste foram criados em caixas plásticas contendo esterco de boi fresco à temperatura de $21 \pm 2{ }^{\circ} \mathrm{C}$. Antes de introduzir os organismos, o pH do substrato foi ajustado até a faixa de 6-7 unidades por adição de carbonato de cálcio. O substrato era renovado e hidratado com água destilada a cada dois meses. Aveia moída era cozida em microondas e servida como alimento uma vez por semana. Os organismos utilizados em ensaio eram sexualmente maduros, da mesma geração, com clitelos totalmente desenvolvidos, e peso entre 300 e $700 \mathrm{mg}$.

O bioensaio agudo com $E$. andrei foi baseado nas recomendações de ASTM (2004). Para tanto, foram utilizados 10 oligoquetas adultos e $500 \mathrm{~g}$ de solo para cada réplica, com total de 4 réplicas, sendo a umidade ajustada 
para 40-60\% da capacidade de retenção máxima de água, com a adição de água destilada. A constituição do solo artificial seguiu as recomendações de OECD (1984), ou seja, $70 \%$ de areia quartzosa, $20 \%$ de caulim e $10 \%$ de pó de fibra de coco, que funcionou como grupo controle. Antes de serem introduzidos no solo, os organismos foram deixados sobre papel filtro umedecido com água destilada em um recipiente de polietileno, durante 24 horas, para o purgamento do conteúdo intestinal. Após esta etapa, cada réplica (i.e., os grupos de 10 minhocas) foi pesada. Durante os 14 dias de exposição em laboratório, os oligoquetas foram mantidos sem alimentação, à temperatura de $22{ }^{\circ} \mathrm{C}$ e com iluminação constante (ASTM, 2004). Ao final do ensaio, os sobreviventes foram submetidos novamente ao purgamento por 24 horas para serem posteriormente pesados. A avaliação da perda de biomassa dos animais (expressa em $\%$ ) foi realizada com base no peso médio dos animais, antes e após a exposição.

A determinação da capacidade de retenção de água (CRA) ou water holding capacity (WHO) foi feita através do procedimento explicitado por Natal-da-Luz et al (2009). O objetivo do teste foi estimar a capacidade de campo de um solo através de um teste simples e rápido para o ajuste da umidade do solos a serem testados com oligoquetas (Figura 1).

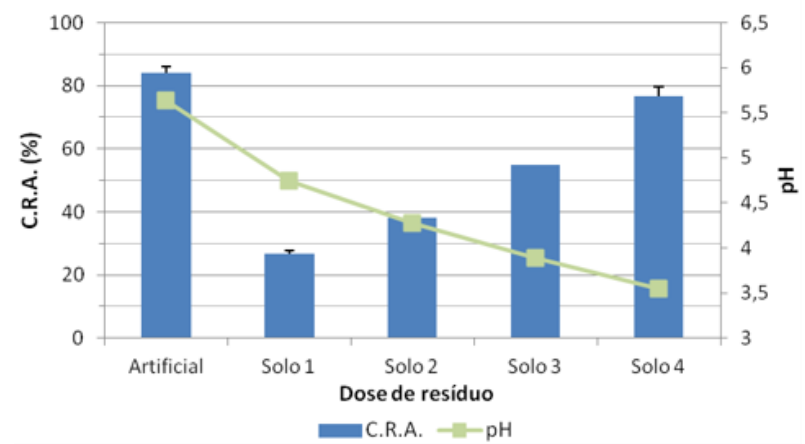

Figura 1. Capacidade de retenção de água e de pH dos 5 solos utilizados no estudo, e barras de erro correspondem a desvios-padrão.
Também foram realizadas análises do $\mathrm{pH}$ de cada solo com o intuito de determinar a alcalinidade ou acidez das amostras de acordo com o proposto pela Embrapa (1997).

\section{Resultados e Discussão}

Com relação ao bioensaio, não foram observados níveis significativos de mortalidade de animais (sempre menor que 5\%), sugerindo baixa toxicidade aguda do solo para os oligoquetas edáficos. Estes dados parecem corroborar, em parte, a indicação do teor de herbicida estipulado pelo MAPA. Porém, como a morte é o mais severo dos efeitos tóxicos não é possível afirmar que a concentração utilizada é segura, sem antes analisar os efeitos referentes à biomassa, reprodução e teste de fuga das minhocas.

Nos solos puros, os maiores níveis de biomassa foram encontrados no solo 3 e no solo 1 , enquanto que os menores níveis foram observados no solo artificial e no solo 4, indicando que as minhocas normalmente preferem solos mais arenosos, porosos, bem drenados e permeáveis (Figura 2).

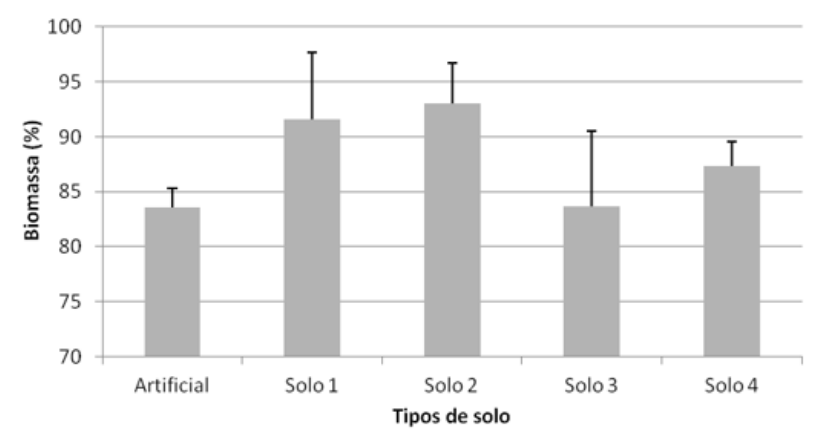

Figura 2. Níveis de biomassa de Eisenia andrei expostas aos solos puros em bioensaio agudo e barras de erro correspondem a desviospadrão.

Para os solos contaminados, houve perda significativa de biomassa para os solos 1 , 3 e 5. Embora tal dose não seja capaz de causar morte, a mesma induziu a perda de peso dos animais, sugerindo a ocorrência de efeitos subletais decorrentes da exposição ao herbicida. A 
maior perda de biomassa aconteceu no solo 1, seguido do solo 5 e que teve perdas similares ao do solo 3 (Figura 3). O solo 1, formado basicamente por quartzo, explica a baixa CRA e a alta toxicidade, tendo em vista a ocorrência potencial da maior concentração de herbicida na solução do solo.

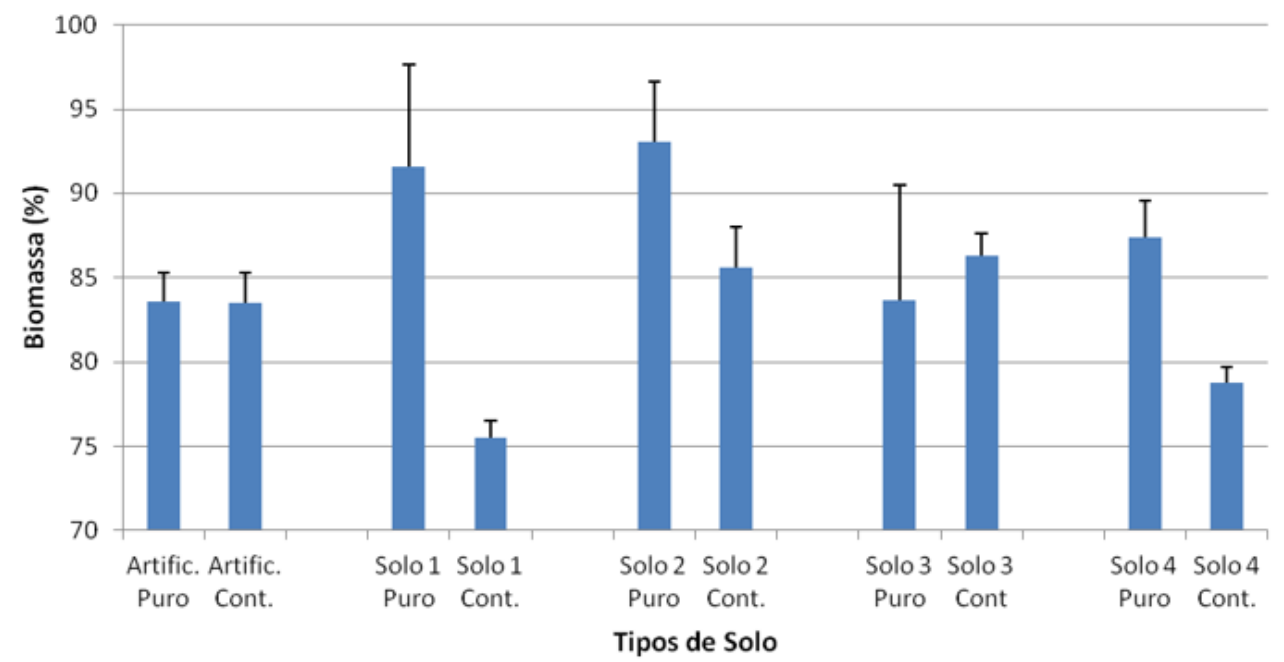

Figura 3. Comparação entre níveis de biomassa de Eisenia andrei expostas aos solos puros e solos tratados com 2,4-D em bioensaio agudo, e barras de erro correspondem a desvios-padrão.

Apesar de possuir maior abundância de assembleias minerais, como a goetita e gibsita que apresentam alto potencial de adsorção de metais e outros contaminantes, devido à sua elevada superfície específica de contato (Vivian et al, 2007; Eberhardt et al, 2008; Cesar et al, 2012; Matzke et al, 2008; Araujo e Melo, 2012), os menores níveis de toxicidade encontrados para o solo 4 sugerem a influência do aspecto textural e da capacidade de sucção do material.

Os resultados obtidos em solos artificiais diferem muito daqueles observados em solos naturais, evidenciando a existência de uma grande lacuna na avaliação ecotoxicológica de solos tropicais, ou seja, a necessidade de se criar um solo artificial que de fato remeta as características das principais classes de solos brasileiros. Na Europa já existem estudos publicados com esta finalidade de simular solos artificiais com as mesmas características dos solos locais (Chelinho et al., 2011)

Apesar de não ser um teste de reprodução, foram observados casulos somente nos solos artificiais, tanto nas amostras contaminadas quanto nas amostras puras, embora não tenham sido quantificadas. Tais resultados sugerem que a performance dos animais em solos artificiais foi melhor em comparação aos solos reais estudados, independente da adição do herbicida.

Além disso, também foi observado que alguns organismos adultos apresentaram alterações morfológicas como fragmentação e perda de segmentos. A ocorrência de tais alterações também foi observada por Nunes (2010). Com isso, pode-se explicar a ocorrência destas alterações em um período curto de tempo, uma vez que a exposição ao agrotóxico é constante.

\section{Conclusões}

O bioensaio mostrou que no solo 4 não havia toxicidade o que sugere como mais provável maior penetração do herbicida em solos devido à alta microporosidade resultando numa maior partição preferencial do 2,4-D para 
o solo, poupando assim a exposição das minhocas.

O teor estipulado pelo MAPA, que define as diretrizes para aplicação do agrotóxico, provoca efeitos subletais para a fauna edáfica e, portanto, é necessário realizar novos estudos, utilizando outros organismos edáficos, para estabelecer o NOEL (non observed effect level). Além disso, é necessário refletir quais os atributos necessários para que o solo artificial reflita a realidade dos solos tropicais. Entre tais características pode-se destacar a presença de oxi-hidróxidos de ferro e alumínio, textura mais argilosa, menores teores de matéria orgânica, abundância de caulinita, entre outros.

Para trabalhos futuros sugere-se a determinação, sob exposição aguda, da concentração letal do 2,4-D em cada tipo de solo estudado, ou seja, determinação da CL50 e do NOEL de letalidade, visando a definição de doses a serem testadas em ensaios de comportamento de fuga, além de definir a CE50 (concentração efetiva a $50 \%$ dos organismos) de biomassa sob exposição aguda.

\section{Referências}

Amarante Júnior, O.P.; Brito, N.M.; Dos Santos, T.C.R; Ribeiro, M.L. Estudo da adsorção/dessorção de 2,4-D em solos usando técnica cromatográfica. Eclética Química, v.27, 2002.

Amarante Junior, O.P.; Santos, Tc.R.; Brito, N.M.; Ribeiro, M.L. Revisão das propriedades, usos e legislação do ácido 2,4diclorofenoxiacético (2,4-D). Cadernos de Pesquisa, v.13, n.1, p.60-70, 2002.

AMERICAN SOCIETY FOR TESTING AND MATERIALS (ASTM). Standard Guide for Conducting Laboratory Soil Toxicity or Bioaccumulation Tests with the Lumbricid Earthworm Eisenia fetida and the Enchytraeid Potworm Enchytraeus albidus. 2004. Disponível em:
<http://www.astm.org/Standards/E1676.htm>. Acesso em: 20 nov. 2014.

Araujo, I.C.L.; Melo, V.F. Sorção de Diuron em Minerais da Fração Argila. Química Nova, v.35, n.7, p.1312-1317, 2012

Carbonell, G., Gómez, J.P.N., Babín, M.M., Fernández, C.; Alonso, E.; Tarazona, J.V. Sewage sludge applied to agricultural soil: ecotoxicological effects on representative soil organisms. Ecotoxicology and Environmental Safety, v.72, p.1309-1319, 2009.

Cesar, R.; Colonese, J.; Silva, M.; Bertolino, L.C.; Castilhos, L.; Egler, S.; Polivano, H.; Bidone, E.; Perez, D. Avaliação da ecotoxicidade de mercúrio em três tipos de solos utilizando ensaios ecotoxicológicos com oligoquetas. Geochimica Brasiliensis, v.24, n.1, p.3-12, 2010.

Cesar, R.G., Silva, M.B., Colonese, J.P., Bidone, E.D., Egler, S.G., Castilhos, Z.C., Polivanov, H. Influence of the properties of tropical soils in the toxicity and bioavailability of heavy metals in sewage sludge-amended lands. Environmental Earth Sciences, v.66, p.2281-229, 2012.

Cesar, R; Sousa, M.A.; Polivanov, H.; Barroso, E.; Alvaro, T.; Colonese, J.; Egler, S.; Castilhos, Z. Disposição terrestre de sedimentos de dragagem: ecotoxicidade, biodisponibilidade de metais e estudo de caso em Belford Roxo (RJ). Geociências, v.33, n.3, p.416-428, 2014.

Chelinho, S.; Domene, X.; Campana, P.; NatalDa-Luz, T.; Scheffczyk, A.; Rombke, J.; Andrés, P.; Sousa, J.P. Improving ecological risk assessment in the Mediterranean area: selection of reference soils and evaluating the influence of soil properties on avoidance and reproduction of two oligochaete species. Environmental Toxicology and Chemistry, v.30, 2011.

Eberhardt, D.N.; Vendrame, P.R.S.; Becquer, T.; Guimarães, M.F. Influência da 
Granulometria e da Mineralogia Sobre a Retenção do Fósforo em Latossolos sob Pastagens no Cerrado. Revista Brasileira de Ciência do Solo, v.32, p.1009-1016, 2008.

EMPRESA BRASILEIRA DE PESQUISA AGROPECUÁRIA (EMBRAPA). Manual de métodos de análise de solo. ed 2 . Rio de Janeiro: EMBRAPA-CNPS, 1997. 212 p.

Gervais, G.; Luukinen, B.; Buhl, K.; Stone, D. 2,4-D Technical Fact Sheet; National Pesticide Information Center, Oregon State University Extension Services, 2008.

Disponível

<http://npic.orst.edu/factsheets/archive/2,4-

DTech.html>. Acesso em 30 out. 2015.

Hinton J.; Veiga, M.M. The influence of organic acids on mercury bioavailability: insight from an earthworm assessment protocol. Environmental Bioindicators, v.3, p.47-67, 2008.

Holt, E.; Weber, R.; Stevenson, G.; Gaus, C. Formation of dioxins during exposure of pesticide formulations to sunlight. Chemosphere, v.88, p.364-370, 2012.

Liu, X.; Chengxiao, H.; Zhang, S. Effects on earthworm activity on fertility and heavy metals bioavailability in sewage sludge. Environment International, v.31, p.874-879, 2005.

Matzke, M.; Stolte, S.; Arning, J.; Uebers, U.; Filser, J. Imidazolium based ionic liquids in soils: effects of the side chain length on wheat (Triticum aestivum) and cress (Lepidium sativum) as affected by different clays and organic matter. Green Chemistry, v.10, p.584591, 2008.

\section{MINISTÉRIO DA AGRICULTURA,} PECUÁRIA E ABASTECIMENTO (MAPA). Consulta de Produtos Formulados, 2003. Disponível em $<$ http://agrofit.agricultura.gov.br/agrofit_cons/p rincipal_agrofit_cons>. Acesso 04 jan. 2014.
Moreira, R.; Sousa, J.P.; Canhoto, C. Biological testing of a digested sewage sludge and derived composts, Bioresour Technology, v.99, n.17, p.8382-8389, 2008.

Nahmani, J., Hodson, M.E., Black, S. A review of studies performed to assess metal uptake by earthworms. Environmental Pollution, v.145, p.402-424, 2007.

Luz, T.N.; Tidona, S.; Jesus, B.; Morais, P.V.; Sousa, J.P. The use of sewage sludge as soil amendment. The need for an ecotoxicological evaluation. Journal of Soils Sediments, v.9, p.246-260, 2009.

Nunes, M.E.T. Avaliação dos efeitos de agrotóxicos sobre a fauna edáfica por meio de ensaios ecotoxicológicos com Eisenia andrei (Annelida, Oligoqueta) e com comunidade natural de solo. 2010. 175 f. Tese em ciências da Engenharia Ambiental Escola de Engenharia de São Carlos, Universidade de São Paulo, São Paulo.

ORGANIZATION FOR ECONOMIC COOPERATION AND DEVELOPMENT (OECD). OECD 207. Earthworm, Acute Toxicity Tests - OECD Guideline for Testing of Chemicals. Paris: OECD, 1984.

Queiroz, J.P.C. Estudo sobre a distribuição do herbicida 2,4-D nos solos da Região de São Pedro da Serra-RJ e sua importância ambiental. 2007. $160 \mathrm{f}$. Tese em engenharia metalúrgica e de materiais - Pontifícia Universidade Católica do Rio de Janeiro, Rio de Janeiro, 2007.

Spadotto, C.A.; Matallo, M.B.; Gomes, M.A.F. Sorção do herbicida 2,4-D em solos brasileiros. Pesticidas: Revista de Ecotoxicologia e Meio Ambiente, v.13, p.103-110, 2003.

Straalen N.M., Donker M.H., Vijver M.G., Gestel C.A.M. Bioavailability of contaminants estimated from uptake rates into soil invertebrates. Environmental Pollution, v.136, p.409-417, 2005. 
Vijver, M.G.; Vink, J.P.M.; Miermans, C.J.H.; Van Gestel, C.A.M. Oral sealing using glue: a new method to distinguish between intestinal and dermal uptake of metals in earthworms. Soil Biology \& Biochemistry, v.35, n.1, p.125-132, 2003.

Vivian, R.; Guimarães, A.A.; Queiroz, M.E.L.R.; Silva, A.A.; Reis, M.R.; Santos, J.B. Adsorção e dessorção de trifloxysulfuronsodium e ametryn em solos brasileiros. Planta Daninha, v.25, n.1, p.97-109, 2007. 